\title{
JUNG LEGGE DANTE
}

TOMMASO PRIVIERO

PHANÊS • VOLUME $2 \cdot 2019 \bullet$ PP. 28-58

https://doi.org/10.32724/phanes.2019.Priviero 


\begin{abstract}
L'articolo presenta in modo del tutto inedito il confronto di Jung con l'opera dantesca, attraverso uno studio diacronico che procede dagli anni giovanili fino agli scritti dei tardi anni '50. Si è inteso seguire passo per passo la cronologia di tale incontro, in cui si intrecciano elementi storici, ermeneutici, e psicologici. Con questo, si è scelto di chiarire il senso generale della lettura dantesca di Jung, obbligati a rimandare la discussione dettagliata dei singoli passaggi menzionati a lavori futuri. Ai fini di proporre un lavoro veramente esaustivo, abbiamo considerato lo spettro completo del materiale disponibile, dalle opere pubblicate a quelle in via di pubblicazione. Per i riferimenti al materiale inedito, e per ulteriore preziose indicazioni, ringraziamo qui Ernst Falzeder, Martin Liebscher, e Sonu Shamdasani, senza cui il completamento di questo articolo non sarebbe stato possibile.
\end{abstract}

\title{
KEY WORDS
}

Psicologia analitica e complessa, C. G. Jung, Dante, ermeneutica, esoterismo. 


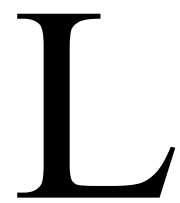

'importanza storica di Dante per Jung è un tema fresco e particolarmente affascinante, su cui poco o nulla è stato scritto. ${ }^{1}$ Diversi autori hanno sì proposto alcune monografie dell'opera dantesca attraverso le lenti della psicologia di Jung, e in particolare H. Luke (1975), P. Sexton (1975), e ancora A. Mazzarella (1991). Tuttavia queste opere, al di là del merito, rientrano in un genere differente, che non considera gli elementi storici o ermeneutici che hanno direttamente avvicinato Jung a Dante, ma piuttosto propone una lettura junghiana del viaggio visionario dantesco. Questa prospettiva presenta due limiti ulteriori. Quello di apparire prima della fondamentale pubblicazione del Liber Novus, e quello di non poter tener conto del materiale inedito di Jung, di cui qui ci si è ampiamente serviti. Più recentemente, D. P. Slattery (2011) ha suggerito una lettura del Liber Novus come la 'Commedia di Jung', ma tale visione, oltre a finire nuovamente per essere fortemente influenzata da una prospettiva junghiana, non si è spinta al livello di un percorso critico approfondito. La lettura che dunque proponiamo qui si sviluppa fin dagli inizi in una direzione divergente rispetto ai lavori precedenti, nel metodo e nel contenuto. Per cominciare, ricordiamo quanto l'elemento più significativo dell'avvicinamento di Jung a Dante sia stato il Liber Novus, ai più noto come il Libro rosso di Jung. In un periodo antecedente al Liber Novus, infatti, i riferimenti a Dante nell'opera dello psicologo svizzero appaiono piuttosto sporadici, mentre proprio nello svilupparsi dell'esperimento visionario che lo occuperà per quasi un ventennio, la figura di Dante, e in particolare la Commedia, diventano un punto di riferimento dei più importanti. Successivamente, Jung si dedicherà alla pubblicazione scientifica e ai seminari con un rinnovato interesse per Dante che l'accompagnerà fino agli ultimissimi scritti. Possiamo così indicare, con una cifra suscettibile di minori correzioni e che esplicitamente non tiene conto né del Liber Novus né dei Libri Neri (richiedenti uno studio apposito), la presenza di più di 70 occorrenze dantesche nell'opera di Jung, tra pubblicazioni scientifiche, seminari, e corrispondenze epistolari. Di questo numero, un paio di occorrenze appaiono prima del Liber Novus, 13 durante, e ben 55 dopo, di cui circa 25 negli anni '30, 21 negli anni '40, e 9 nei ' 50.

${ }^{1}$ Vedi Priviero (2018), Shamdasani (2016). 


\section{IL PRIMO PERIODO (1898-1912)}

Jung legge Dante sin dagli anni giovanili. Difficile dire se lo leggesse già quando sua madre gli suggeriva, all'età di circa 15-16 anni, la sconvolgente lettura del Faust di Goethe (Jung ETG:65). ${ }^{2}$ Difficile anche confermare se la prima giovanile lettura di Jung di Dante avesse avuto o meno lo stesso esplosivo impatto scatenatogli da Nietzsche (Jung 2012:7) o il 'soffio di vita' percepito in Meister Eckhart (Jung ETG:74). Tuttavia sappiamo che già come studente di medicina, Dante figurava nelle letture extracurricolari che Jung voracemente affiancava alla preparazione accademica all'università di Basilea. Qui spendeva il suo tempo tra gli studi, le sedute spiritiche di sua cugina Helene Preiswerk, cui assisteva con alcuni suoi compagni, e gli incontri dell'associazione studentesca Zofingia, di cui diventa presidente nel 1897, tenendo 5 lezioni. In una di queste, nel semestre estivo del 1898, quando compiva 23 anni, il nome di Dante compare per la prima volta ufficialmente nei documenti di Jung (Jung 1983: §229). Dal momento che nel corso degli anni Jung indulgerà in tutti i più importanti simboli danteschi, con una personale predilezione per Lucifero e Beatrice, la menzione di Zofingia appare piuttosto singolare. Jung infatti si serve qui di un episodio noto ma piuttosto specifico della Commedia, ovvero i Canti XXXII e XXXIII dell'Inferno, in cui Dante si figura il Conte Ugolino immerso nel ghiaccio del secondo cerchio (Antenora) della zona più bassa dell'Inferno, riservato ai traditori della patria o del partito (Dante 1987:321).

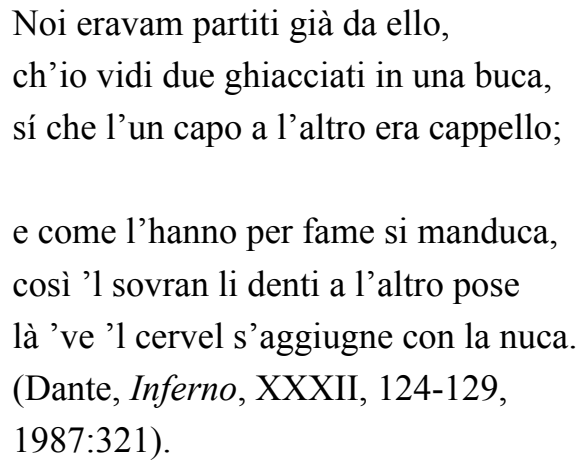

Bisognerà aspettare diversi anni prima che il nome di Dante compaia nuovamente tra le pagine dello psichiatra svizzero. Si tratta della prima versione (1911-1912) di Wandlungen und Symbole der Libido, in

${ }^{2}$ Vedi anche Jung a Max Rychner, 28 febbraio 1932 (Jung Lettere:88). 
cui appare una lunga citazione dalla quarta ecloga di Virgilio, in cui il poeta latino annuncia l'arrivo venturo di un puer salvifico e l'inizio di una nuova età dell'oro (Jung WSL:78). In nota (n3, 78), Jung riprende il fatto che questa ecloga abbia spinto diversi esegeti cristiani a interpretare retrospettivamente Virgilio come poeta quasi-cristiano, annunciatore ante temporem dell'imminente diffondersi del cristianesimo. Questo sembrerebbe esser stato, egli aggiunge, uno dei principali motivi per cui Virgilio assume il ruolo di psicopompo nella Commedia dantesca. ${ }^{3}$ $\mathrm{Al}$ di là di questo collegamento, quel che c'è di più interessante in questa notazione è che da lì a breve sarà Jung stesso, alle soglie delle esperienze visionarie che confluiranno nel Liber Novus, a servirsi di una guida, il vecchio saggio Filemone, le cui caratteristiche finiranno per ricordare molto da vicino il Virgilio dantesco (Shamdasani 2009:202).

\title{
ATTRAVERSO IL LIBER NOVUS (1913-1930)
}

È il 26 dicembre 1913 quando Jung trascrive nei suoi Libri Neri, strato primigenio del Liber Novus, due terzine dal Purgatorio di Dante (Dante 1987:625, 636-637), di cui disponeva in traduzione tedesca (Jung LN:104). ${ }^{4}$

\author{
I' mi son un che, quando \\ Amor mi spira, noto, e a quel modo, \\ ch'e' ditta dentro vo significando. (Dante \\ Purgatorio, XXIV, 52-54). \\ E simigliante poi a la fiammella \\ che segue il fuoco là 'vunque si muta, \\ segue lo spirto sua forma novella. (Dante \\ Purgatorio, XXV, 97-99).
}

Sull'importanza di questo riferimento, e più in generale sulla funzione di Dante nel Liber Novus, rimandiamo all'articolo precedentemente citato.

\footnotetext{
${ }^{3}$ Nella religione greca, psicopompo indica la funzione di chi guida le anime verso il regno dei morti.

${ }^{4}$ La copia della Commedia posseduta da Jung gli era stata regalata da una zia, con dedica, nel 1898. Si tratta di una Göttliche Komödie del 1871-1872, tradotta da F. Notter per Verlag Von Paul (Stuttgart). Il libro presenta segni di lettura frequente, numerose sottolineature e pezzi di carta ad accompagnare pagine contenenti passaggi particolarmente cari a Jung. Un nostro studio delle annotazioni dantesche di Jung è in corso d'opera e di prossima pubblicazione.
} 
Ci limitiamo a ricordare qui che questa duplice citazione testimonia apertamente la rilevanza di Dante per Jung al cuore delle esplorazioni interiori che segneranno questo periodo. Da questo momento in poi, la Commedia dantesca diventa un vero e proprio modello di confronto per il viaggio di Jung, vivificato da una serie di corrispondenze tra l'opera di Dante e il Liber Novus, operanti tanto su un piano letterale quanto simbolico. In questo senso, la primissima lettura dantesca ai tempi dei Libri Neri rivela un carattere fortemente esoterico. Jung non si approccia alla Commedia con intenti critici, lasciando la dimensione letteraria o estetica del lavoro di Dante ben fuori dai propri interessi. Ma nemmeno vi si avvicina con l'intento di un'interpretazione a sfondo psicologico. Vi si rivolge invece in modo meditativo e fortemente pragmatico, nel senso attribuito al termine da William James (1907), partendo dalla convinzione di una profonda corrispondenza tra la propria esperienza visionaria e quella che Dante descrive attraverso i Canti della Commedia. Così, le difficoltà iniziali incontrate di fronte al travolgente materiale psichico in cui si imbatte, lo portano spesso a descrivere le proprie esperienze come una vera e propria discesa agli inferi, o catabasi (Jung LN:237). Qui il ruolo di Dante incomincia a giocare una parte cruciale. Non solo esso infatti rappresenta il modello di una discesa infernale ai tempi del Medioevo, un aspetto che Jung pone al centro del Liber Novus, dichiarando:

\footnotetext{
È necessaria un'opera su cui si possano e si debbano, se occorre, spendere decenni. Devo recuperare una fetta di Medioevo... in me stesso. Abbiamo terminato solo il Medioevo degli... altri. Devo incominciare presto, nel periodo in cui sono scomparsi gli eremiti. [...] Mio caro Io, tu sei un barbaro. Voglio vivere con te, perciò ti trascinerò attraverso tutto l'inferno medievale, finché tu non sia in grado di rendere sopportabile il vivere insieme a te. Dovrai essere vaso e grembo della vita, perciò ti purificherò. Pietra di paragone è l'essere soli con se stessi.

Questa è la via (Jung LN:330, trad. M. A. Massimello 2009).
}

Ma ancora e soprattutto, il modello della Commedia diventa più che altro un riferimento all'anabasi, la risalita dagli inferi, completamento vittorioso della discesa, una psico-cosmologia degli opposti che avvicina, in un modo che William Blake riprenderà, l'energia infernale al rinnovamento psicologico e spirituale simbolicamente rappresentato dalla rinascita nel Paradiso. Dunque, attraverso Dante, il riferimento di Jung alla corrispondenza tra 'fuoco'e 'forma' descrive in modo davvero 
sottile il metodo adottato dallo psicologo svizzero per far fronte alla forza prorompente delle visioni. Si tratta di una combinazione di diversi livelli, e più precisamente del 'fuoco' fornito dalla straordinaria energia psichica del materiale visionario, e della 'forma' data dal tentativo di ospitare queste visioni in uno spazio interiore, 'là vunque si muta', ovvero, qualunque direzione la fiamma delle visioni prenda nel processo di risveglio del fuoco interiore. È bene ricordare che dieci anni più tardi, nel 1923, Jung sosterrà nell'ancora inedito seminario di Polzeath quanto l'unico vero cristianesimo fosse quello medievale (Jung 1923:74). E a questo aggiungerà un'accesa critica della psicologia cristiana precisamente in termini di 'fuoco' $\mathrm{e}$ 'forma', indicando come il più grande limite del cristiano quello di aver perso il fuoco dell'esperienza spirituale, comunicato più di ogni altra cosa attraverso il sesso, l'animalità, e l'immaginazione creativa (Jung 1923:75). Un riferimento come quello della Commedia dantesca, allora, mosso da una profonda ispirazione erotica e modellato sull'immaginazione mistica medievale, si poneva esattamente sul lato opposto di tale impasse.

Quello che preme sottolineare qui, tuttavia, è che oltre il livello esoterico, esiste una seconda, importantissima dimensione della lettura dantesca di Jung di quegli anni. Si tratta del tentativo di affiancare alla lettura diretta di Dante, un'interpretazione della Commedia come un fondamentale esempio di ciò che egli andava delineando in quel periodo nei termini di ermeneutica e psicoterapia 'costruttiva' (Jung 1921, GW6:§701). Vediamo allora i riferimenti danteschi moltiplicarsi. Per cominciare, notiamo che il 15 febbraio del 1918, Jung scrive ad Alphonse Maeder evocando il 'motivo dantesco' per tracciare il 'concetto di anima', Seelenbegriff..$^{5}$ Anticipata in questa lettera, tale prospettiva trova sua completa espressione nei Psychologische Typen, 1921, in cui Dante viene a occupare una posizione di assoluto rilievo nella discussione dei tipi psicologici nella produzione poetica occidentale. I riferimenti a Dante in questo contesto sono tra i più importanti di quelli che si possano trovare nell'intera opera junghiana. È qui, ad esempio, che Jung osserva che la nascita dell'individualismo moderno avrebbe avuto origine con un elemento medievale, l'adorazione della donna, dal momento che 'culto della Donna significava infatti culto dell'anima' (Jung 1921, GW6:\$376). ${ }^{6}$ A questo aggiunge:

\footnotetext{
${ }^{5}$ Corrispondenza Jung-Maeder, 15.02.1918, inedita: 'Ich habe doch Deinen Vortrag expressis verbis anerkannt und die Danteparallele ebenfalls bis auf den Seelenbegriff'. Grazie a Ernst Falzeder per questo riferimento.

${ }^{6}$ Le traduzioni in italiano dalle opere complete di Jung fanno fede a C. G. Jung (19692007). Laddove, come nel caso dei seminari di Jung in inglese, si è voluto suggerire una nuova traduzione italiana, questo è stata indicato a seguito del passo con 'trad. originale'.
} 
Ciò è stato espresso nel modo più bello e più completo nella Divina Commedia di Dante. Dante è il cavaliere spirituale della sua donna; per essa egli supera l'avventura del mondo inferiore e superiore. E in quest'eroica impresa l'immagine di lei si eleva per lui fino a divenire la trascendente mistica figura della Madre di Dio, una figura che s'è staccata dall'oggetto per diventare la personificazione di un fatto puramente psicologico, cioè di quei contenuti inconsci alla cui personificazione io ho dato il nome di Anima. (Jung 1921, GW6:§§376-377).

Accanto a questo passaggio e ad altri in cui ritorna l'immagine di Beatrice, nei Psychologische Typen, anche altri aspetti della Commedia vengono considerati. Particolarmente interessante è la visione per cui secondo Jung la preghiera di San Bernardo nel Canto XXXIII del Paradiso rifletterebbe la trasformazione psichica di Dante, la cui voce è ora innalzata in quella di un santo (Jung 1921, GW6:§§377-378). Centrale e complesso è poi l'accostamento dei contemporanei Dante ed Eckhart, i due grandi maestri dell'anima e della relatività del principio divino nel Medioevo:

Incontriamo in Eckhart idee nuove, idee informate allo stesso orientamento verso l'anima che mosse Dante a seguire l'effigie di Beatrice nel mondo sotterraneo dell'inconscio e ispirò i poeti che cantarono la leggenda del Gral (Jung 1921, GW6:§410).

Da notarsi, infine, è il ripetersi di un riferimento all'episodio del Conte Ugolino (Jung 1921, GW6:§321). Se la presenza dantesca nei Psychologische Typen testimonia complessivamente il crescente interesse diJung per Dante a partire dalla lettura del 1913, troviamo un altro importante riferimento a Dante negli anni '20, che rappresenta in modo evidente la stretta connessione che intercorre tra l'esperienza diretta del Liber Novus e le elaborazioni scientifiche successive. Si tratta del seminario del 1925, in cui Jung decide di commentare (8 giugno 1925) un'importante visione occorsagli nel 1913, precisamente nello stesso periodo in cui trascriveva versi dal Purgatorio, in cui il profeta Elijah riportava il famoso detto ermetico per cui le cose stanno 'come in basso, così in alto', indicando così nella coincidenza degli opposti il motivo supremo dei processi di trasformazione interiore. Nel caso particolare della visione appena citata, il detto si riferiva alla coincidenza riscontrata da Jung tra l'immagine della caverna, in cui ha inizio la penetrazione del profondo, e quella della 
montagna, in cui si sviluppa il rovesciamento e la trasformazione spirituale (Jung LN:252). Jung qui non si dimenticava di ricordare la corrispondenza stabilita da Dante non solo tra gironi infernali e cerchi celesti, ma anche tra la caverna infernale e la montagna purgatoriale, annotando:

Elijah disse che quello che stava sotto era proprio come ciò che stava sopra. Confronta con l'Inferno di Dante. Gli gnostici esprimono la stessa idea nel simbolo dei coni rovesciati.[...] Ne deduco che Dante abbia tratto le sue idee dagli stessi archetipi (Jung 2012:104-105, trad. originale).

I tardi anni '20 sono anche gli anni in cui Jung approfondisce la lettura di Dante con alcuni testi critici. Tra questi, spicca per originalità e importanza il testo per eccellenza degli studi esoterici su Dante del periodo, ovvero Il linguaggio segreto di Dante e dei Fedeli d'Amore di L. Valli (1928), un testo apprezzato da Jung (Jung 1992:102) che era stato in parte tradotto in tedesco per la Europäische Revue ('Die Geheimsprache Dantes und der Fedeli d'Amore') nel 1930. Accanto alla lettura di Valli, che ha permesso di stabilire un'importante connessione tra la lettura dantesca di Jung e l'interpretazione esoterica della Commedia, nella biblioteca di Küsnacht si trovano anche Da Francesca à Béatrice. A travers la Divine Comédie della scrittrice e intellettuale argentina V. Ocampo (1926), il lavoro di H. F. Dunbar, Symbolism in Medieval Thought and Its Consummation in the Divine Comedy (1929), particolarmente annotato da Jung, Mediaeval Culture. An Introduction to Dante and His Time (1929) di K. Vossler, e Dante. L'Antépurgatoire (1944) di G. Méautis. Infine, è ancora in questo periodo che il riferimento dantesco diventa rilevante per Jung anche in materia d'analisi. Così, nel seminario sui sogni del 1928-1930, Jung riporta il sogno di una paziente in cui era apparso il motivo dell'avventura nella foresta oscura (Jung 1984 [1928-1930]:98, n2). Riprendendo la celebre apertura della Commedia (Dante 1987:4344), Jung collega tale immagine onirica alla versione dantesca, dandone il significato di discesa nell'inconscio, partendo dal fatto che nel Medioevo la foresta era l'espressione per eccellenza della compenetrazione tra il sacro, il simbolo materno, e il mistero dei processi di morte e rinascita. ${ }^{7}$

Nel mezzo del cammin di nostra vita

${ }^{7}$ Poco dopo egli notava che era fondamentale osservare come Dante, nel suo viaggio nell'aldilà, si spostava costantemente dall'Inferno al Paradiso, e viceversa. (Jung [192830]:101). 
mi ritrovai per una selva oscura

ché la diritta via era smarrita.

Ahi quanto a dir qual era è cosa dura

esta selva selvaggia e aspra e forte

che nel pensiero rinova la paura!

Tant'è amara che poco è più morte;

ma per trattar del ben ch'i' vi trovai,

dirò de l'altre cose ch'i' v'ho scorte.

(Dante, Inferno, 1-9).

Presumibilmente evocando questi versi, in un certo passaggio del Liber Novus Jung dice di camminare nel mezzo di una foresta oscura e di sentirsi improvvisamente perduto (Jung LN:261). Questa era, si può dire, la situazione in cui Jung si ritrovava a leggere la Commedia di Dante nel momento più critico del suo esperimento visionario, trovando in essa una profonda ispirazione lirica e spirituale. E nella copia della Commedia tedesca da lui posseduta, un pezzo di carta accompagnava la pagina di questo passaggio (Shamdasani 2009:261, n21), quasi a ricordare, come scriveva Hölderlin, che dove cresce il pericolo, cresce anche quello che salva (Hölderlin 1909:347).

\section{LA SVOLTA DEI '30 (1930-1941)}

Quando alla fine degli anni '20 Jung pone fine alla lunga serie di esperimenti immaginativi confluiti nel Liber Novus, ne segue un progetto di studio comparativo che ha lo scopo di trovare fonti contenenti un simbolismo analogo a quello che aveva sperimentato personalmente negli anni precedenti. Troverà il materiale occorrente in due direzioni. $\mathrm{Da}$ un lato, nella tradizione meditativa orientale, e in particolare nello Yoga Sutra di Patanjali, nel Kundalini Yoga, e nel buddhismo tibetano. Dall'altro, nella tradizione esoterica occidentale, espressa per Jung al suo massimo nell'alchimia tardo medievale, ma anche rivelata negli esercizi spirituali di Ignazio di Loyola e in autori visionari quali Dante, Blake, e Nietzsche. È a partire da questa svolta metodologica, e in particolare con riferimento alla seconda categoria, che Dante incomincia a diventare un riferimento costante delle opere e dei seminari negli anni '30.

Nel 1930 Jung dà alla luce Psychologie und Dichtung, un testo in cui elabora a fondo alcuni concetti precedentemente proposti in una lezione 
a Zurigo intitolata 'Über die Beziehungen der analytischen Psychologie zum dichterischen Kuntswerk' (1922). Qui egli propone una distinzione tra una forma creativa 'psicologica' e una 'visionaria' (Jung 1930/1950, GW15:§139). La prima, più ordinaria, lavora con materiale derivante dalla sfera cosciente dell'uomo, traendo ispirazione da quelle emozioni, passioni, ed esperienze che in definitiva rimangono all'interno dei confini di ciò che è comprensibile ed esplicabile da un punto di vista psicologico. La seconda, diversamente, è scatenata da un livello primordiale dell'esperienza immaginativa, in cui tutto è rovesciato, la luce e le tenebre si confondono al di là delle speranze umane di comprensione razionale. Si tratta di visioni, ci viene detto, che non ricordano nulla della vita quotidiana, ma ci trascinano invece nel regno dell'irrazionale, della notte, dei sogni, e delle profondità segrete, magiche e violente, della mente umana (Jung 1930/1950, GW15:\$141). È evidente che nel tratteggiare la dimensione visionaria del processo creativo Jung aveva in mente come punto di partenza le esperienze personali che avevano dato vita al 'più difficile esperimento della [sua] vita' (Jung LN:198), il Liber Novus. Ed è a questo punto che decide di servirsi di alcuni esempi tratti dalla tradizione letteraria occidentale, per meglio chiarire ciò che intende con opera 'visionaria'. Questi esempi rivelano nomi cui Jung ritorna in molteplici occasioni, quasi a formare un personale corpus visionario. Si tratta della seconda parte del Faust di Goethe, dello Zarathustra di Nietzsche, di William Blake, di Jacob Böhme, dell'Hypnerotomachia Poliphili di Francesco Colonna, e, naturalmente, della Commedia di Dante, tra tutti questi uno dei riferimenti preferiti e più ricorrenti di Jung. Di fronte all'opera visionaria, commenta:

Il lettore esige commenti e spiegazioni, prova sentimenti di meraviglia, stupefazione, smarrimento, diffidenza o, quel ch'è peggio, ripugnanza. Qui nulla ricorda la vita quotidiana, qui prendono vita sogni, paure notturne e lugubri intuizioni di tenebre psichiche. La gran maggioranza del pubblico rifiuta una simile produzione, a meno che non parli alle sensazioni più rozze; e davanti ad essa anche lo specialista si trova spesso notevolmente imbarazzato. Dante e Wagner gli hanno bensì reso il compito un po' più facile, poiché nel primo un avvenimento storico e nel secondo un evento mitico ricoprono l'esperienza primigenia e possono quindi passare per "argomento". In entrambi però la dinamica e il significato profondo non risiedono né nel materiale storico né in quello mitico, ma nella visione primigenia che essi esprimono. (Jung 1930/1950, GW15:§§141-143). 
Accanto a due lettere, una del 28 febbraio 1932 e una del 12 dicembre 1938 , in cui Jung riprende Dante scrivendo allo scrittore svizzero Max Rychner e allo storico tedesco Heinrich Zimmer (Jung Lettere:89, 250), sono però soprattutto i seminari ad attestare quanto attraverso tutti gli anni '30 la conoscenza e l'interesse di Jung per Dante si acuiscano notevolmente. Riferimenti a Dante sono infatti presenti nel seminario sul Kundalini Yoga (1932), sulle visioni di Christiana Morgan (1930-1934), a Berlino (1933), sullo Zarathustra di Nietzsche (1934-1939), sui sogni di Wolfgang Pauli nel seminario di Bailey Island e New York (1936-1937), sui sogni dei bambini (1936-1940), nelle lezioni all'ETH (1939, 1940, 1941), e nel seminario sull'interpretazione antica e moderna del sogno (1936-1941).

Per cominciare, nel seminario sul materiale di Christiana Morgan troviamo svariate menzioni all'opera dantesca. Ci sono due riferimenti che più di altro colpiscono la nostra attenzione. Uno è del 24 giugno 1931, in cui Jung s'intrattiene sul significato mistico della rosa nel Medioevo. Qui riprende ancora una volta il prezioso lavoro di L. Valli, Il linguaggio segreto di Dante e dei Fedeli d'Amore, in cui peraltro il filosofo italiano scriveva che la rosa ai tempi di Dante era un simbolo mistico universale, noto 'dall'India fino alle valli della Loira' (Valli 2014:475). ${ }^{8}$ Di questa rosa dantesca, culmine della visione estatica che conclude la Commedia, e che in diverse altre occasioni Jung raffronta ai mandala orientali, egli dice:

La rosa significa amore. E questo rosso è il colore della passione.

Non è un rosso chiaro, ma un rosso denso, un colore infuocato.

Ed è anche il colore della zona muladhara, il centro più basso secondo il Kundalini Yoga. (Jung 1997:413, trad. originale).

Della rosa mistica, insieme al rapporto di Dante con Virgilio, lo psichiatra svizzero si occuperà nuovamente nel seminario di Berlino, ${ }^{9}$ in più di un'occasione nelle lezioni all'ETH (3 marzo 1939, 19 gennaio 1940, 28 febbraio 1941), e nel commento a un'immagine onirica di W. Pauli, parlando della rosa del Paradiso come di un 'mandala cosmico. [...] Un'idea di completa simmetria tra il mondo fisico e quello psicologico' (trad. originale) ${ }^{10}$ Per rimanere invece con le visioni di Christiana Morgan, l'altro riferimento dantesco di rilievo, ripetuto in modo simile in tre occasioni (9 marzo 1932,

${ }^{8}$ Il 7 novembre 1932 Jung scrive a Werner Kaegi a proposito di Luigi Valli (Jung 1992:102).

${ }^{9}$ Di prossima pubblicazione. Grazie a Ernst Falzeder per la segnalazione.

${ }^{10}$ Grazie a Sonu Shamdasani per il riferimento su Pauli. 
14 giugno 1933, 11 ottobre 1933), è quello alla purificazione di Dante attraverso il fuoco, un aspetto i cui risvolti psicologici ed esoterici avevano lungamente occupato Jung durante i suoi esperimenti. Riportiamo il passo:

Per raggiungere una condizione di purificazione, è necessario attraversare quella zona di fuoco in cui ogni desiderio viene bruciato, con il risultato che tutte le ceneri inutili sono spazzate via come dal vento, lasciando soltanto il puro oro che regge il fuoco eternamente. C'è una bella espressione per questo simbolismo nella Divina Commedia di Dante. Nell'ultimo cerchio del Purgatorio, mentre si avvicina alla sfera celeste, Virgilio conduce Dante nella fiamma della purificazione. Lui stesso arretra, poiché in quanto pagano non gli è concesso attraversare la fiamma, ma Dante, battezzato come cristiano, può entrare nel fuoco del puro amore, dove tutto ciò che è terreno viene bruciato, e così finalmente ascendere al cielo. (Jung 1997:1055, trad. originale).

E ancora:

Per Dante fu necessario passare attraverso quella fiamma pura in cui tutto l'agglomerato terreno, il desiderio dell'ego, veniva consumato. Si tratta del fuoco sacrificale, e soltanto colui che è passato attraverso quel fuoco può essere completamente integro e forte e ottenere una condizione suprema. Se si prendono questi stadi come livelli di psicologia, la zona più bassa descritta da Dante corrisponde al muladhara; poi viene la zona mediana, il Purgatorio, che sarebbe la regione del diaframma; e allora la regione superiore sarebbe l'Inferno, in una psicologia o mitologia cristiana; il Purgatorio fu sempre caratterizzato dal fuoco purificatore, che sarebbe la zona manipura. Questo fuoco è l'anticipazione di una condizione di completezza in cui non c'è lesione o dissociazione. Ma nessuno può ottenere questa condizione se non è prima passato attraverso le fiamme del desiderio. (Jung 1997:1107-1108, trad. originale).

L'originale corrispondenza tra il simbolismo del viaggio dantesco e gli stadi del Kundalini Yoga ricorre anche nel seminario dedicato proprio alla Kundalini nel 1932. Qui, ricorrendo al consueto metodo comparativo, prima di tutto evidenzia le somiglianze tra l'inizio dell'Inferno dantesco e il testo medievale dell'Hypnerotomachia. Poi stabilisce un'analogia tra le 
fatiche che i cavalieri del Medioevo intraprendevano per liberare l'Amata, e gli sforzi meditativi degli iniziati a Kundalini (Jung 1999:21). In entrambi i casi si tratta per Jung di simboli di energia femminile rigeneratrice riconducibili all'anima (Jung 1999:22). Più tardi, significativi riferimenti a Dante accompagnano anche il lungo seminario sullo Zarathustra nietzscheano. Lo fanno anzitutto nuovamente in relazione all'immagine dantesca della rosa mistica, simbolo per eccellenza del compimento dell'individuazione e di corrispondenza tra la spiritualità orientale e occidentale. In questo contesto, Jung sviluppa il tema seguendo un'originale analogia con l'esperienza visionaria di Nietzsche ai tempi dello Zarathustra:

Mr Baumann: Dante sperimentò tutto insieme a Virgilio. Erano in due in quel caso.

Dr. Jung: Questo è un buon esempio. [Dante] stabilisce una differenza tra sé e il dynamis, non si identifica con lo psicopompo. Virgilio è per lui certamente lo stesso archetipo, ma il tipo di insegnamento è differente. È il messaggio del Medioevo. Ma l'idea futura è già apparsa quando Dante raggiunge il Paradiso, poiché sulla cima del Paradiso l'individuazione è indicata dalla rosa mistica. Quella è la conclusione del mandala cristiano, la più alta realizzazione di quel tempo, e la rosa mistica è il futuro. Ed è Nietzsche, o Zarathustra, che continua, che riprende il filo eterno e lo conduce più avanti, portando l'idea della rosa mistica dentro l'essere dell'uomo. (Jung 1989:203-204, trad. originale).

Nel seminario su Zarathustra, Jung guarda a Dante anche per introdurre la questione fondamentale di Lucifero e dell'integrazione degli opposti. Qui incomincia a utilizzare una classica immagine dantesca, quella di Lucifero a tre teste, per descrivere il fenomeno della umbra trinitatis, ovvero lo spontaneo manifestarsi di un simbolo psichico di segno opposto a quello della trinità cristiana, volto a un necessario bilanciamento di un'immagine storicamente e psicologicamente troncata a metà. Più avanti, nello svilupparsi delle riflessioni di Jung circa l'esistenza e la consistenza del male, il riferimento al Lucifero dantesco si ripeterà in numerose occasioni, spesso affiancato per analogia a Ecate, dea greca a tre teste protettrice della magia, dei fantasmi, della notte, e della negromanzia.

Uno dei participanti al nostro seminario ha richiamato la mia attenzione sul fatto che nella Divina Commedia di Dante, il diavolo è rappresentato con tre teste. [...] In linguaggio cristiano, 
questo corrisponde alla trinità infernale. (Jung 1989:1084)

Vi ricordate che qualche tempo fa abbiamo parlato della trinità infernale, ovvero del riflesso infernale della trinità celeste, il diavolo tricefalo. Nell'Inferno di Dante esso appare nella forma di Satana, con tre facce, una giallastra, una rossa, e una nera. Ora, da quel momento ho trovato in un trattato medievale un'altra formulazione in cui si dice più che chiaramente che c'è una trinità nel cielo, una nell'uomo, e una all'Inferno. Nietzsche diventa consapevole della trinità all'Inferno dal fatto che percepisce se stesso come una trinità, e tale sentimento gli viene dalla sua identificazione con Dio, la trinità celeste. Negando l'esistenza della divinità cristiana, prima si rende adatto a un processo di inflazione, e poi, per via di una sorta di riflesso a specchio, scopre nuovamente la trinità, ma una trinità in cui egli è incluso. Invece di Padre, Figlio, e Spirito Santo, questa diventa egli stesso, la vita, e la saggezza. (ibid:1172, trad. originale).

Sempre in questi anni, Dante figura anche ripetutamente nel seminario sui sogni dei bambini, in cui ricorrono alcune delle tematiche già delineate, come la funzione purificatrice del fuoco nel Purgatorio dantesco (Jung 2008:188-189), l'archetipo del vecchio saggio attraverso l'analogia Nietzsche/Zarathustra, Dante/Virgilio (Jung 2008:196), e il diavolo tricefalo (Jung 2008:203). Nel seminario sull'interpretazione antica e moderna del sogno, invece, troviamo un riferimento alla rosa come simbolo di totalità psichica (Jung 2014:167) e un interessante passaggio sull'anima:

Come dice Dante, "meditando, percorro il cammino dell'amore". Lì potete trovare la descrizione di come Dante segua la propria anima, ovvero, attraverso l'attesa del suo amore, dell'amore in quanto tale, non per ottenere ricchezze, ma come fine di per sé. Così colui che insegue l'anima come fine di per sè, questa sarà la sua Beatrice, essa diventerà un ponte, un passaggio, perché lei rappresenta una funzione relazionale, una relazione con quello che l'amore è in quanto tale. Ma se lui la ama per la sua dote, o per quello che lei potrebbe rendergli disponibile, costui eliminerà l'eros di torno e lo rimpiazzerà ambiguamente col potere (Jung 2014:200, trad. originale).

In parallelo ai seminari, Dante accompagna in modo significativo 
anche le lezioni tenute all'Eidgenössiche Technische Hochschule (ETH) di Zurigo tra il 1933 e il 1941, prima raccolte in parte da B. Hannah in un'edizione inglese accorciata (1959) e ora in corso di nuova pubblicazione in 8 volumi (di cui il primo, History of Psychology, ha già visto la luce) grazie al progetto della Philemon Foundation. Seguendo cronologicamente il piano di pubblicazione previsto, abbiamo riferimenti a Dante nel volume 6, The Psychology of Yoga and Meditation, con un riferimento alla rosa mistica (3 marzo 1939); nel volume 7, Spiritual Excercises of Ignatius of Loyola, con riferimenti ai simboli del chiostro del re e della regina coeli, e svariate citazioni dai Canti XXXI e XXXII del Paradiso (19 gennaio 1940); nel volume 8, The Psychology of Alchemy, con un'ulteriore menzione alla rosa mistica (28 febbraio 1941), e una alle tre teste del diavolo (4 luglio 1941). ${ }^{11}$ Per quanto riguarda le citate note di B. Hannah sulle lezioni all'ETH, invece, il nome di Dante figura in due contesti ravvicinati, la lezione del 7 giugno 1935 e quella della settimana successiva, il 14 giugno 1935. Nella prima, Jung riprende una prospettiva che aveva già avanzato nel seminario sulla Kundalini, ovvero un parallelo tra alcuni aspetti simbolici della Commedia dantesca e l'Hypnerotomachia di Francesco Colonna. In particolare, ad interessarlo sono ancora l'ingresso nella foresta oscura come il superamento della soglia mentale che conduce nelle profondità dell'inconscio, cui segue per prima cosa l'incontro con gli animali selvaggi (Hannah/Jung 1959:219). È tuttavia nella seconda lezione che troviamo uno dei più interessanti riferimenti a Dante in Jung. Poco prima di avventurarsi in un'analisi del passaggio di Dante sulle gambe di Lucifero come via verso la luce rischiaratrice del Purgatorio, una scena interpretata come esempio di enantiodromia (Hannah/Jung 1959:223-224), lo psicologo svizzero ha appena sottolineato quanto 'si possa imparare davvero tanto da Dante'. E a questo aggiunge:

[Dante] iniziò a scrivere la Divina Commedia quando aveva 35 anni. Il trentacinquesimo anno è un momento di svolta della vita, ed è un fatto interessante che Cristo morì a 34 anni. [...] Un punto esiste, all'incirca al trentacinquesimo anno di età, in cui le cose incominciano a cambiare. È il primo momento del lato oscuro della vita, della discesa verso la morte. È evidente che Dante ha incontrato questo punto e coloro che hanno letto lo Zarathustra sapranno che anche Nietzsche lo scoprì. Quando questo momento di svolta arriva, le persone vi vanno incontro in modi differenti; alcuni ne scappano, altri

${ }^{11}$ Grazie a Martin Liebscher e a Ernst Falzeder per questi riferimenti. 
vi si buttano dentro; e qualcosa di importante accade ad altri ancora dall'esterno. Se non lo riusciamo a comprendere, il Fato lo fa al nostro posto. (Hannah/Jung 1959:223, trad. originale).

Sono molte le ragioni per cui questo passaggio suscita il nostro profondo interesse. Ma tra tutte, viene in mente il modo in cui Jung, circa 20 anni prima, decideva di incominciare il racconto del proprio straordinario viaggio nel profondo in un modo che stabiliva una naturale linea di connessione con le esperienze visionarie dantesche:

\begin{abstract}
Allora, all'età di quarant'anni, avevo ottenuto tutto ciò che mi ero augurato. Avevo raggiunto fama, potere, ricchezza, sapere e ogni felicità umana. Cessò dunque in me il desiderio di accrescere ancora quei beni, mi venne a mancare il desiderio e fui colmo d'orrore. La visione dell'alluvione mi sopraffece e percepii lo spirito del profondo, senza tuttavia comprenderlo. Esso però mi forzò facendomi provare un insopportabile, intimo struggimento, e io dissi: "Anima mia, dove sei? Mi senti? Io parlo, ti chiamo... Ci sei? Sono tornato, sono di nuovo qui." (Jung, 'Il ritrovamento dell'anima', LN:231).
\end{abstract}

\title{
LA FASE TARDA (1941-1959)
}

Con la fine dei seminari dei '30 e le lezioni all'ETH, si apre una fase differente ma altrettanto interessante della lettura dantesca di Jung. Uno degli aspetti principali è certamente quello dell'alchimia. Sappiamo che dal momento in cui Jung riceve da R. Wilhelm una copia del Mistero del Fiore d'Oro (1928), che egli considera un trattato alchemico, l'alchimia acquisirà un ruolo sempre più importante nei suoi studi, sviluppandosi negli anni '30 e poi consolidandosi negli anni a venire. Per quanto siano svariate le connessioni che Jung stabilisce tra Dante e l'alchimia, non è del tutto corretto dire che la scoperta dell'alchimia gli permetta di tornare a Dante secondo una prospettiva, per così dire, alchemica. Al contrario, date le osservazioni fatte finora, è più corretto dire che sia proprio attraverso Dante che Jung giunge a discutere e a comprendere determinati aspetti alchemici tardo medievali.

La connessione tra Dante e l'alchimia suggerita da Jung sembra reggersi su alcune analogie fondamentali. Prima fra tutte, il fatto che tanto il viaggio dantesco per soglie, gironi, e scale celesti, quanto i diversi 
stadi del processo alchemico, come quelli di nigredo, albedo, rubedo, descrivano diverse fasi di iniziazione corrispondenti a una progressiva trasformazione interiore. Il viaggio iniziatico incomincia sempre con il sacrificio dell'identità profana, la morte dell'Io, ovvero il sacrificio degli attaccamenti e dei desideri personali, che in Dante prende forma nella discesa agli inferi e nel linguaggio alchemico è descritta come putrefactio, o putrefazione del sé. Poi, il viaggio prosegue come un processo di graduale rischiaramento interiore o illuminazione, volto a rinfocolare sempre più l'energia vitale ardente sotto le ceneri degli oscuramenti mentali. Infine, il viaggio culmina in un'esperienza di visione e unificazione celeste in cui misticamente non è più possibile distinguere, nella folgorazione della luce, il soggetto che percepisce e l'oggetto percepito, come nell'estatica contemplazione dantesca della rosa celeste alla fine della Commedia. Il secondo elemento di connessione tra Dante e l'alchimia è che lo strumento per eccellenza di tale trasformazione sia l'immaginazione attiva, l'occhio della mente, in grado, come nel caso dell'amore di Dante per Beatrice, di ricongiungere l'amore materiale e l'amore spirituale in un'unica, indivisibile forma di elevazione interiore. Infine, il terzo fondamentale elemento di connessione è che il motivo principale di questa trasformazione sia da cercarsi nell'unione degli opposti, nell'alchimia rappresentata dalla coniunctio del maschile e del femminile, Re e Regina, e in Dante raffigurato dal ricongiungimento divino con l'anima per via dell'amata, Beatrice.

Riportiamo ora alcuni significativi passaggi in merito, tratti dai testi. Già in 'Paracelsus als geistige Erscheinung' (1942), troviamo due riferimenti a Dante. Entrambi riguardano il trattato dell'illustre alchimista svizzero Paracelso, De Vita Longa. La prima menzione, nella sezione su 'Ares', sviluppa nuovamente l'analogia tra l'apparizione del lupo nell' Hypnerotomachia Poliphili e i tre animali selvaggi che Dante incontra nel Canto I dell'Inferno (Jung 1942, GW13:§176n). L' elemento animale rimanda alla forza istintiva e affettiva dell'uomo, senza la cui energia nessun percorso di introversione risulta possibile. Dunque l'animale ha un fortissimo carattere iniziatico. Qui Jung considera, piuttosto singolarmente, la triade dantesca un simbolo analogo alle tre teste di Lucifero. Notiamo curiosamente che questo tipo di analogia non ha praticamente trovato spazio per secoli nella critica dantesca, fino alle originali interpretazioni di Guglielmo Gorni (Gorni 1995, 2008:4). La seconda menzione a Dante nello studio su Paracelso riguarda invece l'apparizione nel De Vita Longa della donna serpente Melusina, che spinge Jung a cercare un raffronto tra la Polia dell'Hypnerotomachia, l'Elena del Faust, e l'immancabile Beatrice dantesca (Jung 1942, GW13:§215). Nello stesso anno, 1942, presentava 
ad Eranos due lezioni, in una delle quali, 'Der Geist Mercurius', notava una significativa differenza tra la visione dantesca e quella alchemica:

Gli alchimisti lo hanno perfino qualificato come unità trina, per dimostrare con ciò la sua perfetta corrispondenza con Dio. Come si sa, in Dante Satana è tricefalo, quindi una trinità nell'unità. Satana è una corrispondenza di Dio, ma come antitesi. Non è affatto questa la concezione degli alchimisti: essi vedono in Mercurio un'emanazione armonica con l'essenza di Dio o la sua creazione. (Jung 1942, GW13:§283).

Nei primi anni '40, nel frattempo, registriamo anche due richiami a Dante nella lezione di Jung sull'Aurelia di Gérard de Nerval (Jung 2015:52,90). Sempre a proposito dell'alchimia, invece, ricchi e variegati sono i rimandi a Dante in Psychologie und Alchimie (1944). Troviamo qui anzitutto svariate immagini rappresentanti episodi della Commedia dantesca: un acquerello di Blake (The Ascent of the Mountain of Purgatory, 1824-7) in cui si vede Dante in rosso guidato nell'ascesa del monte del Purgatorio da Virgilio in blu, erroneamente frainteso in didascalia da Jung come l'anima o Beatrice (Jung GW12, fig. 19). Ancora Dante e Virgilio che scendono nell'aldilà, da una raffigurazione del 1400 contenuta nel Codex Urbanus Latinus 365 (Jung GW12, fig. 69). Dallo stesso Codex, una rappresentazione della rosa celeste, il fiore cosmico (Jung GW12, fig. 83). E poi riferimenti di contenuto, di cui il più interessante ricorre in una digressione sulla nekyia, in cui le narrazioni apocrife della discesa di Cristo agli inferi vengono associate con il materiale dantesco, una corrispondenza che gioca una parte particolarmente importante per la stessa nekyia di Jung ai tempi del Liber Novus:

Nekyia, vєxvía da véxuৎ (cadavere), titolo dell'undicesimo canto dell'Odissea, è il sacrificio funebre per evocare dall'Ade i defunti. Nekyia è dunque un termine adatto per il "viaggio nell'Ade", la discesa nel mondo dei morti, ed è usato in questo senso anche da Dieterich (1913) nel suo commento al Codice di Akhmim, che contiene un frammento apocalittico del vangelo di Pietro. Esempi tipici sono la Divina Commedia, la "Notte di Valpurga classica" del Faust, i racconti apocrifi delle discese all'inferno di Cristo ecc. (Jung 1944, GW12:§61, n2).

Giusto un anno più tardi, nel 1945, Jung pubblicherà la prima 
versione del suo saggio 'Der philosophische Baum' in Verhandlungen der Naturforschenden Gesellschaft Basel, poi rivista e notevolmente ampliata in una successiva versione nel 1954 in Von den Wurzeln des Bewusstseins: Studien über den Archetypus. Anche in questo testo Jung non manca di servirsi di Dante in due occasioni piuttosto particolari (Jung 1945, GW 13:§389, 410n). In una di queste, in un passaggio sul simbolo alchemico dell'albero rovesciato, immagine riflessa e rovesciata dell'Albero della Vita cui alludono molteplici tradizioni, dal buddhismo alla Kabbalah, Jung rimanda in nota a un momento non eccessivamente considerato del Purgatorio dantesco, il Canto XXII, vv. 131-138 (Dante 1987:612-613). Dante, Virgilio e Stazio stanno ormai procedendo nella sesta cornice del Purgatorio, quando i loro 'dolci ragionamenti' sono improvvisamente interrotti dall'apparizione di un albero a metà strada. Un albero, per l'appunto, rovesciato, dai cui rami pendono frutti profumati.

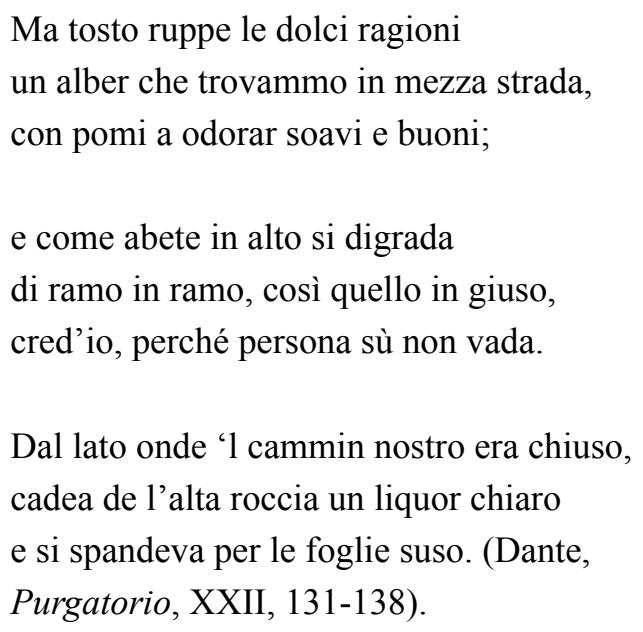

Notiamo che oltre a Jung, questa visione ha affascinato anche A. Coomaraswamy, che ne ha scritto tracciando paralleli con testi sacri indiani (Coomaraswamy 1987:340-341). Per quanto riguarda l'altra occorrenza dantesca in 'Der philosophische Baum', essa è parte di un passo sulla rosa mistica che menziona anche i Fedeli d'Amore, di cui Jung aveva appreso nel dettaglio dal testo di Valli:

Nell'ambito spirituale la rosa, in quanto rosa mystica, appartiene, come l'hortus aromatum e l'hortus conclusus, alle allegorie di Maria; il suo significato mondano è invece quello della donna amata, è la rosa dei poeti, $\mathrm{i}$ "fedeli d'amore" del tempo. Maria è allegorizzata da san Bernardo come "medium terrae", da Rabano 
Mauro come "civitas", dall'abate Goffredo di Admont come "castellum" e come "domus divinae sapientiae", e da Alanus de Insulis (Alain de Lille) come "acies castrorum". Analogamente, anche la rosa ha il significato di un mandala, come è evidente nella rosa celeste del Paradiso dantesco. [Come nel suo equivalente, il loto indiano, la rosa è decisamente femminile.] In Matilde di Magdeburgo la rosa va intesa come proiezione dell'Eros femminile sulla persona di Cristo. (Jung 1945, GW13:§389).

Ancora dieci anni più tardi, a conferma di una ricerca diffusa sulle analogie tra principi alchemici e Dante, Jung riprendeva Dante anche nell'ultimo dei suoi studi sulle relazioni tra psicologia e alchimia, Mysterium Coniunctionis (1955/1956), con un riferimento particolare alla catabasi che dà avvio al processo di purificazione dell'alchimista e che Dante avrebbe sperimentato nella sua discesa agli inferi (Jung 1955/1956, GW14:\$493).

Accanto a quanto detto sull'alchimia, c'è certamente un altro aspetto da evidenziare in questa sezione riguardante la fase più tarda dell'interessamento di Jung per Dante. Questo èil moltiplicarsi di riferimenti alla raffigurazione dantesca di Satana a tre teste, già citata varie volte anche in quest'articolo. Ecco la gloriosa apparizione di Lucifero nel Canto XXXIV dell'Inferno (Dante 1987:334-335), già anticipata da Virgilio a inizio Canto con una parafrasi che modifica l'inno di Vanenzio Fortunato alla croce, 'Vexilla regis prodeunt', con l'aggiunta di una sola terrificante parola, 'inferni', così da ottenere 'si avvicinano i vessilli del Re . . dell'Inferno':

Lo 'mperador del doloroso regno

da mezzo 'l petto uscìa fuor de la ghiaccia;

e più con un gigante io mi convegno,

che i giganti non fan con le sue braccia:

vedi oggimai quant'esser dee quel tutto

ch'a così fatta parte si confaccia.

S'el fu sì bel com'elli è ora brutto,

e contra 'l suo fattore alzò le ciglia,

ben dee da lui proceder ogne lutto.

Oh quanto parve a me gran maraviglia

quand'io vidi tre facce a la sua testa!

L'una dinanzi, e quella era vermiglia; 
l'altr'eran due, che s'aggiugnieno a questa

sovresso 'l mezzo di ciascuna spalla,

e sé giugnieno al loco de la cresta:

e la destra parea tra bianca e gialla;

la sinistra a vedere era tal, quali

vegnon di là onde 'l Nilo s'avvalla.

Sotto ciascuna uscivan due grand'ali, quanto si convenia a tanto uccello: vele di mar non vid'io mai cotali.

Non avean penne, ma di vispistrello era lor modo; e quelle svolazzava, sì che tre venti si movean da ello:

quindi Cocito tutto s'aggelava.

Con sei occhi piangea, e per tre menti gocciava 'l pianto e sanguinosa bava.

Da ogne bocca dirompea co' denti un peccatore, a guisa di maciulla, sì che tre ne facea così dolenti.

A quel dinanzi il mordere era nulla verso 'l graffiar, che talvolta la schiena rimanea de la pelle tutta brulla. (Dante, Inferno, XXXIV, 28-60).

Il Satana di Dante deve davvero aver colpito Jung, comunque se lo sia immaginato da questa lettura. Oltre che nei sopracitati richiami nei seminari, lo troviamo in Die Psychologie der Übertragung (Jung 1946, GW16:§403). Di nuovo in 'Versuch zu einer psychologische Deutung des Trinitäsdogmas' (1948), al cuore di questa riflessione:

Il volere cosa diversa e opposta caratterizza il diavolo, come la disobbedienza caratterizza il peccato originale in genere. Come già si disse, son queste le premesse per la creazione e devono quindi essere iscritte nel piano divino e con ciò incluse nella sfera divina. Ma la definizione cristiana di Dio come summum bonum esclude 
il maligno fin dall'inizio, mentre secondo il Vecchio Testamento egli era pur stato uno dei figli di Dio. Così il diavolo, come simia Dei, rimase fuori dell'ordinamento trinitario e in antitesi con esso. Alla rappresentazione del Dio trino con tre teste corrispose un Satana tricefalo, come appare, per esempio, in Dante. Con ciò, analogamente all'Anticristo, si allude a un'antitrinità infernale, a una vera umbra trinitatis. (Jung 1948, GW11: §252).

E ancora in 'Zur Phänomenologie des Geistes im Märchen' (Jung 1948, GW9i:§425), nel contesto di un raffronto tra la trinità ctonia che gli alchimisti pongono a completamento di quella celeste e il Lucifero dantesco. Come questi riferimenti testimoniano ripetutamente, la questione non era affatto secondaria. Infatti Jung si serve del diavolo dantesco principalmente come un esempio vibrante della metà mancante della Trinità cristiana, l'altra faccia di Dio che l'occidente avrebbe cercato di rigettare nel nome di un'illusoria scissione psichica tra summum bonum e infimum malum. Al contrario per Jung, la via verso l'unione degli opposti passa anzitutto attraverso l'integrazione del diavolo, poiché, come scriveva Blake nel Marriage of Heaven and Hell, il diavolo è energia, e senza energia non c'è movimento degli opposti, né progressione psicologica e spirituale (Blake 1906:7). Allora, che a rappresentare tale integrazione ci sia anzitutto il 'Re dell'Inferno' di Dante, non può passare inosservato. Come ha infatti brillantemente raccontato Marina Montesano nel suo 'The Devil's Hellish History: Satan in the Middle Ages' (Montesano 2018), è proprio attraverso il Medioevo che il diavolo acquisisce tutt'un'altra identità in Europa, da angelo caduto o gregario perduto di Dio, a vero e proprio archetipo di sacralità negativa, degno di uno statuto paritario a quello divino. Su questa base, il viaggio di Dante nella Commedia offre un modello perfetto per il senso che Jung attribuisce all'integrazione del diavolo. Dante entra nel suo viaggio percorrendo un sentiero, la voragine infernale, formatasi dalla caduta di Lucifero al centro della terra. Sarà soltanto attraversando passo per passo la strada aperta dall'Anticristo, che Dante sarà infine in grado di superare e oltrepassare il gigantesco mostro satanico per avviarsi verso il primo passo di un rovesciamento mentale radicale. Anche Ellenberger, riprendendo il suo concetto di 'malattia creativa', scrive al riguardo:

Un elemento caratteristico di ogni viaggio attraverso l'inconscio è il verificarsi di ciò che Jung ha chiamato enantiodromia. Questa parola, che risale a Eraclito, significa "ritorno all'opposto". Certi processi mentali si trasformano a un certo punto nei loro 
opposti, come ad opera di una sorta di auto-regolazione. Questa nozione è stata esemplificata simbolicamente dai poeti: nella Divina Commedia vediamo Dante e Virgilio che raggiungono il punto più profondo dell'Inferno, e iniziano poi da lì il primo passo verso l'alto, in opposta direzione verso il Purgatorio e il Paradiso. Questo misterioso fenomeno di spontaneo capovolgimento della regressione è stato sperimentato da tutti coloro che sono passati con successo attraverso una malattia creativa, ed è diventato un aspetto caratteristico della terapia sintetico-ermeneutica junghiana (Ellenberger 2018 [1970]:826).

Il diavolo tricefalo riflette dunque l'elaborazione di Jung circa il problema della realtà del male, un aspetto cruciale del suo pensiero che se a livello pratico era già profondamente emerso nel Liber Novus, a livello teoretico trova forse le sue più importanti riflessioni in una fase tarda, culminante in Aion (1951) e Antwort auf Hiob (1952).

A conclusione del nostro percorso, occorre infine segnalare che anche gli anni '50 vedono l'interesse di Jung per Dante espandersi in diverse direzioni. Nelle riflessioni elaborate a proposito del simbolismo dei mandala ('Über Mandalasymbolik', 1950) non poteva mancare un cenno esoterico alla rosa mistica, considerata l'equivalente occidentale del loto buddhista e posta a fianco del fiore d'oro cinese e della rosa dei Rosacroce (Jung 1950, GW9i:§652). Nel 1952, scrivendo la premessa al Lucifero e Prometeo di Werblowsky, ricordava poi quanto poco fosse disposto a leggere il materiale visionario della Divina Commedia in chiave psicologica (Jung 1952, GW11:§468). Due anni più tardi, Dante compare nuovamente in 'Das Wandlungssymbol in der Messe' (1954), in un parallelo tra le schiere scintillanti dei Santi nelle visioni del Paradiso e la ricorrenza archetipica del 12 derivante dallo zodiaco (Jung 1954, GW11:§424). Nel 1958, riprendeva in una discussione al Club Psicologico di Basilea la funzione di Virgilio come psicopompo della catabasi dantesca (Jung 1993:373). Infine, l'ultimissima citazione da Dante presente negli scritti di Jung è in un'introduzione al testo di Brunner, Die Anima als Schicksalsproblem (Jung 1959, GW18: 1279n), in cui figura un rimando al celebre testo di $\mathrm{H}$. Corbin, L'Imagination créatrice dans le Soufisme d'Ibn 'Arabī, nel quale si comparavano le esperienze d'amore di Dante e dei Fedeli d'Amore con quelle dei poeti mistici persiani:

La giovane dama che era per Ibn 'Arabī alla Mecca quello che Beatrice era per Dante, era una vera donna, per quanto fosse al 
tempo stesso una figura teofanica "in persona", la figura della Sophia aeterna (che alcuni dei compagni di Dante chiamavano Madonna Intelligenza). (Corbin 1997:100, trad. originale).

\section{CONCLUSIONI}

Lo scopo generale di quanto presentato è stato introdurre il lettore al percorso, particolarmente ricco e complesso, che ha portato Jung verso Dante. Come la pubblicazione del Liber Novus nel 2009 e la prossima apparizione dei Libri Neri hanno messo in luce, questo avvicinamento occupa una posizione nuova e di tutto rilievo nell'opera dello psicologo svizzero. Dobbiamo infatti ricordare che mentre alcuni aspetti dello studio di Jung, come il confronto con Nietzsche (Bishop 1995; Parkes 1999; Liebscher 2001, 2003, 2012; Domenici 2019) o con Goethe e il romanticismo (Bishop 2007 / 2008, 2011), rivelino ormai una letteratura particolarmente consolidata, il rapporto con Dante è ancora tutto da scoprire, nel solco del profondo valore psichico che Jung attribuiva al Medioevo e alla tradizione visionaria occidentale. In questa direzione, per quanto in uno spazio purtroppo contenuto, abbiamo soprattutto voluto evidenziare che esistono diversi livelli che legano Jung a Dante, e fondamentalmente un livello esoterico, che forse potremmo anche chiamare psicologico, in un senso profondo, e uno ermeneutico. Il primo consiste in quella particolare lettura di Dante che ha accompagnato le esperienze straordinarie contenute nel Liber Novus, risultanti in corrispondenze tanto letterali quanto simboliche. Ci siamo visti costretti a rimandare ad altra sede la discussione approfondita di questo livello, per quanto esso contenga per esperienza diretta tutto quanto Jung elabori a livello critico su Dante negli anni successivi al Liber Novus. Il secondo livello riguarda invece l'utilizzo della Commedia di Dante da parte di Jung come di un esempio eccezionale di quello strato mentale visionario che costituisce per lui la base di una forma di psicoterapia, e di ermeneutica, costruttiva e non-riduttiva. Se l'importanza di Swedenborg per Jung in questa direzione è già stata vagliata (Ellenberger 1970, Taylor 2007, Shamdasani 2016), quella di Dante, che ha ispirato entrambi, è ancora da affrontare in profondità. Abbiamo potuto osservare che da un punto di vista storico questi due livelli si intrecciano sviluppandosi secondo diverse fasi, rispettando un generale incrementarsi dell'interesse per Dante e una significativa ricorrenza tematica, che vede nel diavolo, nella rosa mistica, e 
nel 'ritrovamento dell'anima' i capisaldi della discussione dantesca di Jung.

University College London

tommaso.priviero.15@ucl.ac.uk

\section{ABBREVIAZIONI}

$\mathrm{ERG}=[1962]$. Erinnerungen, Träume, Gedanken von C. G. Jung. Aufgezeichnet und herausgegeben von Aniela Jaffé. Zürich / Düsseldorf: W. Verlag.

$\mathrm{WSL}=1925$ [1911 / 1912]. Wandlungen und Symbole der Libido.

Beiträge zur Entwicklungsgeschichte des Denkens. Leipzig / Vienna:

Franz Deuticke.

$\mathrm{GW}=[1995]$. Gesammelte Werke 1-20. Ostfildern: Patmos Verlag.

$\mathrm{LN}=$ Liber Novus $=[$ Das Rote Buch] The Red Book: Liber Novus. Ed. e intr. di Sonu Shamdasani, traduzione di Mark Kyburz, John Peck, e Sonu Shamdasani. New York / London: W.W. Norton \& Company.

Lettere $=2015$ [1973]. C. G. Jung Letters, selezionate ed edite da Gerhard Adler, in collaborazione con Aniela Jaffé, traduzione di R. F. C. Hull. Princeton: Princeton University Press.

\section{BIBLIOGRAFIA}

Alighieri, Dante. 1871-1872. Göttliche Komödie. Tr. F. Notter. Stuttgart: Verlag Von Paul.

_. 1987[1306/1321]. Commedia. Milano: Garzanti.

- 2011. Opere. Rime, Vita Nova, De vulgari eloquentia. Milano: Mondadori.

- 2014. Opere. Convivio, Monarchia, Epistole, Egloghe. Milano: Mondadori.

Bishop, Paul. 1995. The Dionysian Self: C. G. Jung's Reception of Nietzsche. Berlin: Walter de Gruyter. 
—. 2007 / 2008. Analytical Psychology and German Classical Aesthetics: Goethe, Schiller, and Jung. Vol. 1 e 2. London: Routledge.

- 2011. Reading Goethe at Midlife. Ancient Wisdom, German Classicism, and Jung. New Orleans, LA: Spring Journal Books.

Blake, William. 1906[1790/1793]. The Marriage of Heaven and Hell. Boston: J. W. Luce and Company.

Boccassini, Daniela. 2018. 'Earthly Paradise: Dante's Initiatory Rite of Passage'. Temenos Academy Review 21: 61-92.

—. 2019. 'Down to the Father's Womb: Jung's and Dante's Encounters with the Dead'. Jung Journal: Culture \& Psyche 13(2): 46-81.

Coomaraswamy, Ananda. 1987. Il grande brivido. Saggi di simbolica e arte. Milano: Adelphi.

Corbin, Henry. 1997[1958]. Alone with the Alone. Creative Imagination in the Sufism of Ibn 'Arabī. Princeton: Princeton University Press.

Domenici, Gaia. 2019. Jung's Nietzsche. Zarathustra, The Red Book, and 'Visionary Works'. London: Palgrave Macmillan.

Dunbars, Helen Flanders. 1929. Symbolism in Medieval Thought and Its Consummation in the Divine Comedy. New Haven: Yale University Press.

Ellenberger, Henri. 2018[1970]. La scoperta dell'inconscio. 2 vol. Milano: Bollati Boringhieri.

Gorni, Guglielmo. [1995]. 'Lettura del primo canto della Commedia'. In Simone Invernizzi (a cura di). Esperimenti Danteschi. Inferno 2008. Genova-Milano: Marietti, 2008.

Hannah, Barbara. 1959. Notes on Lectures Given at the Eidgenossische Technische Hochschule, Zurich by Prof. Dr. C. G. Jung. Zurigo: stampa privata.

Hölderlin, Friedrich. 1909. Gesammelte Werke. Jena: Eugen Diederichs. James, William. 1922[1907]. Pragmatism. A New Name for Some Old Ways of Thinking. New York: Longman, Green, and Co. Jung, Carl Gustav. 1983[1897/1899]. The Zofingia Lectures.

Supplementary, Volume 1. London: Routledge.

- 1917[1911/1912]. Psychology of the Unconscious. A study of the 
Transformations and Symbolisms of the Libido. New York: Moffat, Yard \& Company.

_ 2009[1913/1929]. The Red Book: Liber Novus. Ed. and intr. Sonu Shamdasani, trad. Mark Kyburz, John Peck, and Sonu Shamdasani. NewYork/London: W.W. Norton \& Company.

—. [1918]. 'Corrispondenza Jung-Maeder'. (Inedito).

—. [1921]. Psychologische Typen. Gesammelte Werke von C. G. Jung, vol. 6 .

—. [1922]. 'Über die Beziehungen der analytischen Psychologie zum dichterischen Kuntswerk. Gesammelte Werke von C. G. Jung, vol. 15.

-. [1923]. Notes on the Seminar in Analytical Psychology. Conducted by Dr. C.G. Jung. Polzeath, England, July 14 - July 27, 1923. Arranged by Members of the Class. (Inedito).

- [1925]. Introduction to Jungian Psychology: Notes of the Seminar Given by Jung on Analytical Psychology in 1925. Edizione originale edita da W.McGuire, edizione revisionata ed. da Sonu Shamdasani. Princeton: Princeton University Press, 2012.

—_ [1928/1930]. Seminar On Dream Analysis. Ed. William McGuire. Princeton: Princeton University Press, 1984.

—. [1930/1950]. Psychologie und Dichtung. Gesammelte Werke C. G. Jung, vol. 15.

—. [1930/1934]. Visions: Notes of the Seminar Given in 1930-1934 by C. G. Jung. Ed. Claire Douglas. Princeton: Princeton University Press, 1997.

- [1932]. The Psychology of Kundalini Yoga. Notes on the Seminar Given in 1925 by C. G. Jung. Ed. Sonu Shamdasani. Princeton: Princeton University Press, 1999.

. [1933]. The Berlin Seminar. Ed. Giovanni Sorge, tr. Mark Kyburz and John Peck. Princeton: Princeton University Press. (Inedito).

- [1934/1939]. Nietzsche's Zarathustra: Notes of the Seminar Given in 1934-1939. Princeton: Princeton University Press, 1989.

- [1936/1937]. Dream Symbols of the Individuation Process: Notes of the Seminars given by Jung in Bailey Island and New York, 1936-7 Ed. Suzanne Gieser. Princeton: Princeton University Press. (Inedito).

- [1936/1940]. Children's Dreams. Notes from the Seminar 
Given in 1936-1940. Ed. Maria Meyer-Grass e Lorenz Jung. Tr. Ernst Falzeder con la collaborazione di Tony Woolfson, 2008.

- [1936/1941]. Dream Interpretation Ancient and Modern. Notes from the Seminar Given in 1936-1941. Princeton: Princeton University Press, 2014.

- [1938/1939]. The Psychology of Yoga and Meditation. In Modern Psychology: C. G. Jung's Lectures at the ETH Zürich, 1933-1941. Ed.MartinLiebscher. Tr.Heather McCartney eJohn Peck. Vol.6(Inedito).

- [1939/1940]. Spiritual Exercises of Ignatius of Loyola. In Modern Psychology: C. G. Jung's Lectures at the ETH Zürich, 1933-1941. Ed. Martin Liebscher. Tr. Caitlin Stephens. Vol. 7 (Inedito).

- [1940/1941]. The Psychology of Alchemy. In Modern Psychology: C. G. Jung's Lectures at the ETH Zürich, 1933-1941. Eds. Christopher Wagner e Martin Liebscher. Vol. 8 (Inedito).

- [1942]. Paracelsus als geistige Erscheinung. Gesammelte Werke von C. G. Jung, vol. 13.

—. [1942b]. Der Geist Mercurius. Gesammelte Werke von C. G. Jung, vol. 13.

- [1942c]. On Psychology and Visionary Art. Notes From C. G. Jung's Lecture on Gérard De Nerval's Aurélia. Ed. Craig E. Stephenson. Princeton: Princeton University Press, 2015.

—. [1944]. Psychologie und Alchimie. Gesammelte Werke von C. G. Jung, vol. 12.

—. [1945/1954]. Der philosophische Baum. Gesammelte Werke von C. G. Jung, vol. 13.

—. [1946]. Die Psychologie der Übertragung. Gesammelte Werke von C. G. Jung, vol. 16.

- [1948]. Versuch zu eitner psychologische Deutung des Trinitäsdogmas. Gesammelte Werke von C. G. Jung, vol. 11.

—. [1948b]. Zur Phänomenologie des Geistes im Märchen. Gesammelte Werke von C. G. Jung, vol. 9.

—. [1950]. Über Mandalasymbolik. Gesammelte Werke von C. G. Jung, vol. 9.

- [1952]. 'Foreword to Werblowsky's Lucifer and Prometheus'. 
Gesammelte Werke von C. G. Jung, vol. 11.

. [1954]. Das Wandlungssymbol in der Messe. Gesammelte Werke von C. G. Jung, vol. 11.

—. [1959]. 'Foreword to Brunner's Die Anima als Schicksalsproblem'. Gesammelte Werke von C. G. Jung, vol. 18.

__. 1969-2007. Opere complete 1-18. A cura di Luigi Aurigemma. Milano: Bollati Boringhieri.

—_. 1993[1977]. Jung Speaking. Ed. William McGuire e R. F. C. Hull. Princeton: Princeton University Press.

—. 2015[1973]. C. G. Jung Letters. Selezionate ed edite da Gerhard Adler, in collaborazione con Aniela Jaffé, traduzione di R. F. C. Hull. Princeton: Princeton University Press.

Jung, Carl Gustav / Jaffé, Aniela. 1995[1962/1983]. Memories, Dreams, Reflections. Tr. Richard and Clara Winston. London: Fontana Press.

Liebscher, Martin. 2001. 'Jungs Abkehr von Freud im Lichte seiner Nietzsche-Rezeption'. In Zeitenwende-Wertewende. Ed. Renate Reschke. Berlin: Akademic-Verlag.

—. 2003. 'Die 'unheimliche Ähnlichkeit.' Nietzsches Hermeneutik der Macht und analytische Interpretation bei Carl Gustav Jung'. In Rüdiger Görner and Duncan Large (eds.). Ecce Opus. Nietzsche Revisionen im 20. Jahrhundert. London / Göttingen: Vandenhoeck \& Ruprecht: 37-50.

—. 2012. Libido und Wille zur Macht. C. G. Jungs Auseinandersetzung mit Nietzsche. Basel: Schwabe.

Luke, Helen. 1975. From Dark Wood to White Rose. Journey and Transformation in Dante's Divine Comedy. New Mexico: Dove Publications.

Mazzarella, Adriana. 1991. Alla Ricerca di Beatrice. Milano: IN/OUT.

Montesano, Marina. 2018. 'The Devil's Hellish History: Satan in the Middle Ages'. National Geographic History (October).

Ocampo, Victoria. 1926. Da Francesca à Béatrice. A travers la Divine Comédie. Madrid: Revista de Occidente.

Parkes, Graham. 1999. 'Nietzsche and Jung: Ambivalent Appreciations'. In Jacob Golomb, Weaver Santaniello, and Ronald Lehrer (eds.). 
Nietzsche and Depth Psychology. Albany: SUNY Press: 69-213.

Priviero, Tommaso. 2018. 'On the Service of The Soul. C.G. Jung's Liber Novus and Dante's Commedia'. Phanês: Journal for Jung History 1: 28-57.

Sexton, Patricia Mary. 1975. The Dante-Jung Correspondence. California: Joyce Motion Picture Co.

Shamdasani, Sonu. 2009. 'Introduzione' a The Red Book: Liber novus. Ed. and intr. di Sonu Shamdasani, trad. Mark Kyburz, John Peck, and Sonu Shamdasani. New York/London: W.W. Norton \& Company.

- 2016. 'Descensus ad Infernos: la saison en enfer de C.G.Jung'. In Danger et necessité de l'individuation: 9e Colloque de Bruxelles. Brussels: Esperluète Eds.

Slattery, Dennis Patrick. 'Thirteen Ways of Looking at the Red Book. C. G. Jung's Divine Comedy'. Jung Journal: Culture and Psyche $5(3): 128-144$.

Taylor, Eugene. 2007. 'Jung on Swedenborg, redivivus'. Jung History 2(2): $27-31$

Valli, Luigi. 1904. Il fondamento psicologico della religione. Roma: Loescher.

- [1922]. Il segreto della Croce e dell'Aquila nella Divina Commedia. Milano: Luni Editrice, 2015.

—. 1925. La chiave della Divina Commedia. Bologna: Zanichelli. . [1928]. Il linguaggio segreto di Dante e dei Fedeli d'Amore. Milano: Luni Editrice, 2014. 